\title{
LA PERMEABILIDAD ENTRE LOS ORDENES ECLESIÁSTICOS Y LA INSTAURACIÓN DEL ORDEN DE LOS LAICOS EN CASTILLA Y EN EL REINO-IMPERIO LEONÉS (1000-1075)
}

\author{
POR
}

\author{
Dolores Mariño VerRas
}

Universidad de Cantabria

\begin{abstract}
RESUMEN
La permeabilidad y ambivalencia estructural de los ordenes eclesiásticos se evidencia en los conjuntos arquitectónicos que confornan las sedes episcopales hasta el úlimo tercio del s. XI. Estas agrupaciones horizontales de templos y monasterios, adyacentes o inmediatos a la Iglesia principal, facilitan la pervivencia de la trabazón funcional señalada.
\end{abstract}

\begin{abstract}
Amstract
The permeability and the ambivalence structural of the ecclesiastic orders is very clear in the architectural sets of the episcopal sees until the last third of the $1 \mathrm{t}^{\text {th }}$ century. These horizontal shrine groups and monasteries, adjacent or immediate to the principal Church, facilikate the survive of the functional way indicated.
\end{abstract}

Castilla y el reino-imperio leonés están inmersos en las corrientes europeas de pensamiento que especulan hacia mediados del s.XI sobre la función eclesiástica y secular, y sobre las relaciones de colaboración entre las distintas potestades. En el transcurso del segundo tercio del s. XI los ordenes eclesiásticos se restauran y se refuerza el carácter sagrado de los elementos y personas vinculadas con Dios. Nuevas solidaridades cohesionan a monjes y clérigos 
frente al orden secular que aparece de forma tácita entre los años 1055-1063 con el fin de cerrarlo a los eclesiásticos.

Hasta entonces, el conjunto de personas más distinguidas de la sociedad cristiana, en especial las que ostentan una supremacía moral y una autoridad sagrada, se agrupan en órdenes racionalmente estructurados, que son permeables para sus integrantes y cerrados para los des-ordenados, es decir, para quienes no pertenecen a orden alguno, o son «REFUGANOS» que lo abandonaron y no pueden reincorporarse sin dar muestras ostensibles de arrepentimiento.

\section{AMBIVALENCIA Y PERMEABILIDAD ENTRE LOS ÓRDENES ECLESIÁSTICOS}

Las dos grandes instituciones ascéticas de la Cristiandad se definen lenta y paulatinamente a partir del último tercio del $\mathrm{s}$. XI, puesto que las diferencias existentes ente el «ORDO MONASTICUS» y el «ORDO CLERICORUM» corresponden más a criterios morales que a grupos propiamente funcionales. De ahí que el primero absorba competencias sacerdotales durante toda la centuria' ${ }^{1}$ q que perviva la figura del monje-sacerdote ${ }^{2}$, cuya ambivalencia funcional acaso prolonga creencias antiguas sobre la superioridad moral de los monjes para administrar los sacramentos. Por ello, durante la primera mitad de la centuria, afluyen con naturalidad regalos y generosidades a manos de los abades por el ejercicio de su «SACERDOTIO»", así como donaciones hacia monasterios donde se realizan las «CONFESIONEM» $\mathrm{y}$ «SACRATIONEM» ${ }^{4}$.

El propio sínodo-imperial de Coyanza admite que tanto clérigos como monjes pueden bendecir nupcias ${ }^{5}$ y conminar a la penitencia aun cuando su-

I En el año 1114, los obispos de las sedes gallegas y de Oporto se reunen para promulgar, entre otros cánones, el que dispone «QUE LOS MONJES VIVAN BAJO LA OBEDIENCIA DEL ABAD, NO TENGAN NINGUNA PROPIEDAD Y NO EJERZAN OFICIOS PÚBLICOS COMO LOS PÁRROCOS» Historia Compostelana, Ed. E. FALQUE, Madrid, 1994, pp. 240-241.

2 «MONACUS PALATII : MAGISTER ASCARIUS ET PRESBITER» (Año 1020, RUIZ ASENCIO, J.M. Colección documental de la Catedral de León, III, León, 1987, doc. 581 pp. 97-101). «MONACUS QUI SACERDOTIUM AMISERUNT...ET QUI SUA HORDINEM TENENT» (Año 1012, Ibidem, 707, 263-265). La unión de «ABBA ET SACERDOS» en una persona perdura durante el iltimo tercio del s. XI (Año 1080, FlORIANO LLORENTE, P. Colección diplomática del monasterio de S. Vicente de Oviedo, I, Oviedo, 1968, doc. LXXXUl, pp. 152-153).

3 En el año 1021 el abad Vimara dona bienes que proceden «DE MEA MUNIFICIENTIA IN MEO SACERDOTIO DE MEIS DECIMIS ET ECCLESIIS» ( RUIZ ASENCIO, J.M. Col...León, III, $776,358-359$ ).

4 Affo 1009, RuIz ASENCIO, J.M. Col... Leon, III, 677, 220-223. Ibidem, IV, 905, 15-16.

5 Decr. V, 4 y V, 2 de ambas redacciones. Ed. GARCiA GALLo, A. El Concilio de Coyanza. Contribuciones al estudio del Derecho Canónico español en la Alta Edad Media, Madrid, 1951, p. 24.

$\mathrm{I}^{\text {er }}$ Congreso de Historia de la Iglesia Hispania Sacra 51 (1999) 
ponga la expulsión de la Iglesia para los pecadores no contritos por haber cometio determinadas faltas o delitos ${ }^{6}$.

Los abades reciben su «ORDINATIONEM» y cooperan «AD ORDINANDUM» a los monjes después de procurarles la instrucción necesaria ${ }^{7}$. Dirigen un «COLEGIO DE CLERIGOS O MONJES», o un «CORO DE MONJES, FRATRUM Y CLERIGOS ${ }^{8}$ que están capacitados para acoger a una turbamulta de sacerdotes, obispos, monjes, «REFUGANOS»... y «A CUALQUIER PERSONA CONVERSO DE CADA ORDEN ${ }^{9}$. Entre dichos «CONVERSOS» figuran presbíteros y clérigos acogidos al régimen o potestad del abad o del prepósito a quien encomiendan sus personas ${ }^{10}$. A pesar de las recomendaciones que sobre lo contrario existen desde antiguo, abundan los casos de presbíteros y diáconos que son «ABBAS» o «FRATER» de su comunidad sin abandonar el grado y responsabilidades clericales ${ }^{H}$. Y durante la segunda mitad del s. XI perduran las ordenaciones presbiterales de monjes que se mantie-

La denominación de sínodo-imperial fue utilizada por CH. J. BISHKO «Fernando I y los orígenes de la alianza castellano-leonesa con Cluny», C.H.E., XLVII-XIVIII, 1968, p.82.

6 Y ello de acuerdo con los «sagrados cánones». Ver el decr. IV, 1,2 de la redac. conimbricense de Coyanza (Ed. García Gallo, cit. p. 23). Garcla Gallo reconoce el confusionismo existente entre clérigos y monjes en esta versión portuguesa (Ibidem pp. 100-101).

7 El abad Domingo de Rozuela « LITTERA DOCUIT ET ORDINEM ACCEPIT ET VITAM DOXIT» (Año 1033, RuIz ASENClO, J.M. Col... León, IV, 918, pp. 30-31). Los abades deben lograr que los monjes memoriecen el Salterio "AD ORDINANDUM» a éstos (redac. conimbricense de Coyanza, V [3], Ed. GARCla GALLo, p. 24). Asimismo, tienen encomendada la «SCHOLAM ET DISCIPLINAM» de las «Iglesias canónicas» y la dirigen «AD EPISCOPOS CLERICOS ORDINANDOS» (Cap. II, [2] del Concilio Compostelano de 1056, Ed. MARTínEZ DfEZ «El Concilio Compostelano del reinado de Fernando L» A.E.M., 1, 1964, p. 128).

8 «ABBA VEL VESTRO COLLEGIUM CLERICORUM VEL MONACHORUM» del monastrio leonés de S. Martín de Valdepueblo (Año 1033, RUZZ ASENCIO, J.M. Col... León, IV, 916, 28-29), «COLLEGIO MONACHORUM, SACERDOTUM, DIACHONORUM VEL CONFESORUM » (Año 1022, HERRERO DE LA FUENTE, M. Col... Sahagún, II, 410, pp. 57-58) ; «CORUM MONAGORUM, FRATRUM, CLERICORUM» (Año 1039, HERRERO DE LA FUENTE, Col... Sahagún, II, 455, pp. 113-114).

9 Año 996, RuIZ ASENCIO, J.M. Col... León, III, 574, 88-90, en copia de la segunda mitad del s. XI. Se denomina conversos a los clérigos arrepentidos de levar una «VITAM LAICALEM» a finales del s. XI (Año 1095, $O$ tombo de Celanova, I, Ed. J.M. ANDRADE, Santiago, 1995, n ${ }^{\circ} 43$, p. 73). La experiencia eremítica del primer tercio del $\mathrm{s}$. XI no excluye la vida claustral y facilita el desarrollo del movimiento de penitentes y el cenobitismo.

10 Año 1013, Herrero de la FuENTE, M. Col... Sahagún, II, 400, pp. 44- 46; año 1034, RuIZ ASENCIO, J.M. Col... León, IV, 925, 39-41.

"I Ello ocurre a lo largo del s. XI. Está bien documentado el caso del «FRATER» y presbítero Teodomiro que fue abad del monasterio de Santiago de León (Año 1011, RuIZ ASENCIO, J.M. Col... León, III, 700, pp. 250-252; año 1015, Ibidem, 736, 301-302...). El presbítero Fernando fue abad de S. Andrián hasta después de la integración de dicho monasterio en la sede episcopal leonesa, acaecida entre 1059-1081 (RuIZ ASENCIO, Col... León, IV, 115, 323-24; 1120, 492-494...). 
nen en el orden monástico si cuentan con el preceptivo consentimiento del obispo $^{12}$.

El «ORDINE CLERICORUM» integra a presbíteros, diáconos y subdiáco$\operatorname{nos}^{13}$. Su ritual de ordenación es divulgado por los libros «ORDINOS» ${ }^{14}$ que lo singulariza ${ }^{15}$ entre los tradicionales «SEPTEM GRADIBUS» de la jerarquía de oficios eclesiásticos. Atienden Iglesias y monasterios, rurales o urbanos, del sistema familiar o propio; así como decanías ${ }^{16}$ de las que son «TENENTES» por disposición episcopal desde mediados del s. XI. Intervienen activamente en su gestión patrimonial, perciben aranceles por actos espirituales ${ }^{17}$, y tienen posibilidades de incrementar sus bienes personales gracias a la conmutación de sanciones disciplinarias, e incluso carcelarias, cuando recaen sobre monjes fugitivos o «REFUGANOS» ${ }^{18}$. La sociedad cristiana de la primera mitad del s.XI tolera que presbíteros y diáconos lleven una vida secularizada con mujer e hijos $^{19}$, y que sus costumbres y aspecto no difieran de los laicos en el gusto común por las armas, la caza, las barbas y el pelo largo...20.

12 Año 1071, RUIZ ASENClO, J.M. Col...León, IV, 1180, p. 423

13 Véase la restauración de la sede episcopal de Palencia por Vermudo III (Año 1035, ABAJO MarTin, T. Documentación de la Catedral de Palencia (1035-1047), Palencia, 1986, doc. 1, pp. 3-5). Precisamente su primer obispo Poncio había sido antes monje en Ripoll y abad en Tabémoles. Durante el primer tercio del s. XI el «CLERIGATUM HORDINE» aparece generalmente mencionado en copias documentales de tines del s. XI o en Tumbos y Cartularios (LuCAS ALVAREZ, M. El Tumbo de San Julián de Samos (siglos VIII-XII), Santiago de C., 1986, doc. 5-9, pp. 454-456). En el segundo tercio del s. XI abundan los textos apócrifos o de dudosa fiabilidad que interpolan párafos sobre aspectos del «MORE ROMANOw, investidura de cargos, procedimientos de elección... propios de épocas posteriores.

14 Aparecen entre los habituales que poseen muchas Iglesias y monasterios. Los presbíteros Froila y Didago reciben en 1060 un «LIBER ORDINO, ALIOS II MISTIGOS, PSALTERIO I» (RUIZ ASENCIO, J.M. Col...León, IV, 1118, pp. 327-328)

15 Sin embargo no figura en el LIBER COMMICUS, según señalan los editores de este (PÉREZ DE URBEL, F.J. y GONZÁLEZ Y RUIZ-ZORRILA, A., I, Madrid, 1950, p. CXXIV).

16 Durante la primera mitad del s. XI su intervención en las decanf́as aparece generalmente recogida en Tumbos y Cartularios. Año 1032, O Tombo de Celanova Ed. J.M. ANDRADE, I, 35, pp. 64-66.

17 Año 1016, RuIZ ASENCIO, J.M. Col...León, III, 742, pp. 311-312.

18 Que tenían bajo su encomendación por mandato episcopal (Año 1052, RUIZ ASENCIO, J.M. Col...Le6n, IV, 1088, pp. 284-285). Las Leyes de León se hab́an opuesto a los monjes errantes, y otorgaron poder a los obispos sobre los monjes y monjas de su diócesis. Ver decr. 3 y III de ambas versiones Ed. PÉREZ PRENDES «La potestad legisltiva en el Reino de León» Reino de León en la Alta Edad Media, I, Cortes, Concilios y Fueros, León, 1988 pp. 528 y 533.

19 «EGO IOHANNES CLERICI ET UXORI MEA GOTA AD VOBIS PATRI NOSTRO VEILA ABBA...» donan bienes y una tierra «DE NOSTRO FLLIO ANDRES PRESBITER» (Afio 1039, RUIZ ASENCIO, J.M. Col...León, IV, 983, pp. 129-130)

20 No son infrecuentes los casos de presbíteros que mueren por heridas de guerra, o de obispos asesinados. Ruiz ASENCIO, J.M. Col...León, IV, 957, 85-87; QUINTANA PRIETO, A. Crisis de la Iglesia astorgana en el s. XI, Astorga, 1971, pp. 107 y 139.

$\mathrm{I}^{\text {er }}$ Congreso de Historia de la Iglesia Hispania Sacra 51 (1999) 
Los presbíteros y diáconos alcanzan la «DISCIPLINA» de su grado junto a los obispos ${ }^{21}$, a quien tienen por «DOMNO» y le prestan «SERVITIUM» y «OBEDIENTIAM» como fieles suyos ${ }^{22}$. Se promocionan con facilidad a las dignidades episcopal ${ }^{23}$ y de «PRIMICLERICI», y además a los cargos de «MAIORDOMO» 0 «MAIORDOMO DE RECULA» sin que suponga desdoro a la fe monacal de quienes la profesan. Simultanean distintos oficios clericales: «PRESBITER... PRIMICERIUS ET CANTOR MAIOR»; «PRESBITER, MAIORDOMO ET PRIMICLERUM $»^{24}$ que armonizan con el abacial durante todo el s. $\mathrm{XI}^{25}$.

Las sedes episcopales se componen de un «COLLEGIO MONACHORUM» 0 de una corporación de monjes y clérigos que llevan vida monástica y son destinatarios de donaciones ${ }^{26}$. Aunque allí residan otros obispos ${ }^{27}$, sólo uno preside las «REGULAS SEDIBUS SUIS» ${ }^{28}$ y gobierna en colaboración

21 Placencio fue ordenado presbítero «ANTE EPISCOPUS», recibiendo el nombre de Sabater (Año 1025, HERRERo de LA FUENTE, M. Col...Sahagún, II, 416, pp. 65-66). Año 1037, Ruiz ASENCIO, J.M. Col...Le6́n, IV, 957, pp. 85-87. Los retratos ideales del obispo ensalzan su labor en la formación religiosa de los clérigos, desde S. Gregorio Magno (en su «Regla Pastoral», P. RICHÉ, La Educación en la cristiandad Antigua, Barcelona, 1983, p. 29) basta los obispos reformadores del s. XII.

22 Año 1034, RUIZ ASENCIO, J.M. Col..León, IV, 934, pp. 50-52; año 1037, Ibidem, 949, 70-71...

23 Es habitual la cooptación de obispos entre presbíteros y diáconos. Véanse los casos de los obispos Nuño y Servando de León (RuIZ AsENClo, J.M. Col..León, III, 635, 171-172; 754, 327 329...); Pedro Kendulfiz y Sampiro de Astorga (RU1z ASENCIO, Ibidem, pp. 865, pp. 473-475); Pelayo Sisnandiz y Diego Peláez de Compostela (Núñez ConTRERAS «Colección Diplomática de Vermudo III, rey de León», H.I.D., 4, 1977, doc. n²0, pp. 494-496).

24 Año 989, RUIZ ASENCIO, J.M. Col...León, II, 527, pp. 24-25; año 1003, 635, pp. 171-172; año 1012,710 , pp. $271-272$..

25 Véase el ejemplo del presbítero y «PRIMICLERO» Ordoño Rodríguez, que era abad del monasterio de S. Félix de León (Año 1092, Ruiz ASENClO, J.M. Col..Leôn, IV, 1272, 574-575; 1275, p. $579 ; 1302,623-624 \ldots$... Dicho monasterio había sido fundado por el obispo leonés D. Nuño, y estuvo vinculado a sus «SOCIORES ET CONGERMANAS» y al episcopado (Año 1020, RUIZ ASENCIO, J.M. Col...León, II, 770, pp. 349-351; Ibidem, IV, 935, pp. 52-53...)

26 Se hacen donaciones «PRO SUSTENTATIONE MONACORUM VEL CLERICORUM IBI HABITANTIUM» (Año 1047, RUIZ ASENCIO, J.M. Col..León, IV, 1048, pp. 224-226). Los «CLERICI» de la sede leonesa reciben «SUSTANTIAM TEMPORALEM» del monarca a través del obispo (Año 1049, Ibidem, 1067, pp, 252-253).

27 Acogidos en calidad de posibles obispos «IN PARTIBUS», jubilados... con capacidad para atender algún oficio litúrgico en alguno de los templos y monasterios que constituyen las sedes. Sobre la dualidad de prelados en algunas sedes, ver A. PALOMEQUE TORRES, Episcopologio de las sedes del reino de León, León, 1966, pp. 40,44,48... Sobre los obispos «IN PARTIBUS» cuyas diócesis están afectadas por el poder musulmán ver Z. GARCf́a VIllaDA, Historia Éclesiástica de España, III, Madrid, 1936, pp. 244, 252; y PALOMEQUE TORRES, Ibidem, p. 13.

28 «EPISCOPUS PONTIUS IN REGULAS SEDIBUS SUIS» (Año 1032, HERRERO DE LA FUENTE, M. Col...Sahagún, II, 433, pp. 87-88). Don Cipriano de León era obispo «IN SEDIS SANCTE MARIA VIRGINIS HIC IN REGULA» (Año 1053, RUIZ ASENCIO, J.M. Col...Leon, IV, 1090, pp. 286-287). «EPISOCPUS PELAGIUS IN REGULA SANCTE MARIE» (Año 1067, loidem, 1147, pp. 376-377). 
con la comunidad eclesiástica colegiada ${ }^{29}$. Cuando aceptan el «ORDEN REGULAR» ${ }^{30}$, se rigen por diferentes reglas y costumbres que son unificadas en Coyanza bajo el «ORDINEM SANCTI ISIDORI VEL BENEDICTI ${ }^{31}$.

La ambivalencia, permeabilidad y complementariedad estructural entre los órdenes eclesiásticos se evidencia en los conjuntos arquitectónicos que conforman las sedes episcopales hasta finales del s. XI. León, Oviedo, Santiago de Compostela... están constituídas por una agrupación horizontal de templos y monasterios adyacentes e inmediatos a la Iglesia principal ${ }^{32}$, que facilitan la pervivencia de la trabazón funcional entre clérigos y monjes hasta el s. XII.

\section{DEFINICIÓN DEL ORDEN ECLESIÁSTICO E INSTAURACIÓN DEL ORDEN SECULAR}

La definición del orden eclesiástico implica la identificación de los elementos sagrados, y la caracterización de las personas eclesiásticas por una moral sexual, costumbres y una función propia en el seno de la comunidad cristiana.

Durante el segundo tercio del s. XI los elementos y personas vinculados con Dios y con las cosas divinas o santas se refuerzan en su carácter sagrado ${ }^{33}$.

29 "AEPISCOPUS CUM OMNIUM MONACORUM IBI VITAM DEGENTES" (Año 991, RUIZ ASENCIO, J.M. Col...León, III, 550, 55-58); «AEPISCOPO... UNA PARITER CUM COLLEGIO MONACORUM DE SEDE SANCTE MARIE» (Afío 1032, Ibidem, Col...León, IV, 899, 6-8). «PELAGIO EPISCOPO SEU ETIAM VEL CLERICI QUI IBIDEM SUNT PERMANENTES QUAM ET QUI HIC PERMANSERINT ET VITAM MONASTICAM DEDUXERINT» (Afio 1066, RUIZ ASENCIO, J.M. Col...León, IV, 1144, 370-372). Al igual que los pesbíteros, estos obispos pudieran tener atribuciones abaciales, que son conocidas en zonas próximas. PÉREZ DE URBEL, F.J. Sancho el Mayor de Navarra, Madrid, 1950, p. 311.

30 Abades y presbíteros de $S$. Vicente de Oviedo proclaman su pertenencia al «RECULAREM ORDINEM» en el año 1048. FLORIANO LLORENTE, P. Colección diplomática... cit. XLIV, pp. 97-99.

${ }^{11}$ En su redacción conimbricense, puesto que la ovetense relega el «orden de S. Isidoro» en pro de la singularidad benedictina. Decr. II [1], Ed. A. GARCÍA GALLO, El Concilio... p. 18.

32 En tomo a la Iglesia de Santa María de León se instalaron los monasterios de Santiago, S. Vicente, S. Miguel, S. Pelayo, Santos Pedro y Pablo, S. Juan Bautista, S. Félix... En Oviedo, junto a la Iglesia del Salvador, estaban los monasterios e Iglesias de S. Vicente, S. Pelayo, Santa Cruz, Santa Marina... H. RODRÍGUEZ BALBIN, De un monte despoblado a un fuero real 700 a 1145. Estudio sobre los primeros siglos del desarrollo urbano de Oviedo, Oviedo, 1977, p. 428. En Santiago de Compostela estaban agrupados cuatro Iglesias y dos monasterios: Santiago, S. Salvador, S. Juan y la Iglesia del monasterio de S. Esteban F. LOPEZ ALSINA La ciudad de Santiago de Compostela en la Alta Edad Media, Santiago, 1988, pp. 246-247 y 263-264.

33 «CONFIRMO PER DEUM ET DIVINA HOMNIA OUE SUNT SACRA» (Año 1039, RUIZ ASENClO, J.M. Col...León, IV, 974, pp. 119-120; año 1044, Ibidem, 1012, pp. 178-179...). Refiriéndose a tiempos pasados, Pelayo Conde se acusa de haber cometido actos criminales o impios «CONTRA

$I^{\text {er }}$ Congreso de Historia de la Iglesia Hispania Sacra SI (1999) 
LOS «DEXTROS» se delimitan ${ }^{34}$ como espacio donde los «REFUGANOS» y los laicos tienen prohibido convivir con mujeres y poseer bienes o derechos ${ }^{35}$. Junto a las Iglesias son lugares de refugio para los acusados de cualquier delito $^{36}$, y objeto de acciones reivindicativas variadas que pretenden la restitución de su integridad jurídica en manos eclesiásticas ${ }^{37}$.

También los «MINISTERIA» destacan su sacralidad o santidad ${ }^{38}$, y se detallan sus componentes: cruces, cálices, cofres... ${ }^{39}$ para adscribirlos al orden eclesiástico y excluirlos del circuito de intercambios. Obispos, presbíteros, diáconos, o cualquier otro ministro de la Iglesia, tienen prohibido comprar o vender «IPSA SACRA MINISTERIA, NEC OLEUM, NEC ULLA DE ORDINE ECCLESIASTICO», so pena de simonía y reputación de falsos cristianos $^{40}$. El consorcio de canónigos compostelanos dictamina, por consiguiente, que sólo pueden lucrarse de los «BENEFICIA ECCLESIAE» quienes pertenezcan a alguno de los «SEPTEM GRADIBUS» de la jerarquía de oficios ${ }^{41}$.

DEUM IN SANCTIS SUIS...ID EST : MONASTERIOS DEVASTVI REBUS SANCTAE ECCLESIAE CONTEMPNI...DOMINUM MEUM ATQUE PONTIFICEM MORTI TRADEREM...» (AĨo 1076, QUINTANA PRIETO, A. Crisis de la Iglesia astorgana... cit., doc. XII p. 139 y p. 107).

34 Los treinta pasos de época visigótica, reconocidos en la versión ovetense de Coyanza, (decr. XII 1, Ed. GarCía GaLLo p. 28) se amplian a setenta y dos por el Concilio Compostelano de 1056 (cap. III, [1] , Ed. G. MARTíNEZ DíEZ «El concilio compostelano... cit. p. 128.

35 Decr. III [16] de ambas redacciones de Coyanza (Ed. GARCía Gallo p. 22); cap. 1H, 11] del Concilio Compostelano de 1056 (Ed. MARTINEZ DfEZ p. 128).

36 Decr. XII, 1 de Coyanza (Ed. GARCía Gallo p. 28). Con posterioridad, en el s. XII, los obispos excluyen de esta protección a los esclavos, ladrones, traidores, excomulgados, monjes desertores y a quienes violen Iglesias (c. II del Concilio de Oviedo de 1115, Ed. TEJADA Y RAMRRO, J. Colecciones de Cánones y de todos los Concilios de la lglesia de España y América, II, Madrid, 1855, pp. 39-44).

37 Mediante recursos judiciales contra los posesores de derechos limitativos de estas propiedades eclesiásticas. Véase el pleito interpuesto por el obispo de Lugo en el año 1078 contra los condes que pretenden conservar bienes en los «DEXTROS DE ILLA SEDE SANCTAE MARIAE LUCENSIS», Risco, M. España Sagrada, XL, (1796); pp. 417-422.

38 LOS «MINISTERIA SANCTA» exigen que los oficiantes posean un aspecto cuidado (cap. VI [3] del Concilio Compostelano de 1063, Ed. MARTíNEZ DíEZ, p. 131) además del grado e instrucción convenientes.

39 «NODICIA DE ILLA MINISTERIA QUE IBI RECEPIMUS, IT EST, CRUCE I ET CAPSA ET CALICE DE ARGENTO, LIBER ORDINO, ALIOS IIOS MISTIGOS, PSALTERIO I', CUPAS Llas, CUPOS Ilos». Fueron entregados en el año 1060 a dos presbíteros (RUiz ASENCiO, J.M., Ibidem, $1118,327-328$ ). El Libro Iudiciorum y el Fuero Juzgo se ocuparon de las transacciones y títulos de los bienes de la Iglesia (Ver las leyes del Tit. I, Libro V Ed. RAE).

40 Cap. II, [3] del Concilio Compostelano de 1056 (Ed. MARTÍNEZ DIEZ, cit. p. 125). El Concilio Compostelano de 1063 especifica que «IPSA SACRA MINISTERIA, NEC PRO ORDINATIONE, NEC PRO BAPTISTERIO, NEC PRO NULLA ORDINE ECCLESIASTICA" cap. II, [4] (Ed. MARTÍNEZ DíEZ, Ibidem).

4l Cap. V de dicho Concilio de 1063 (Ed. MARTínez DíEZ, p. 129). 
A partir de 1055 los eclesiásticos se singularizan en la sociedad cristiana por la castidad ${ }^{42}$, la prohibición de llevar $\operatorname{armas}^{43}$ y por su aspecto: vestido talar, tonsura visible y barba rasurada ${ }^{44}$. Ésta resulta indispensable para tocar los «MINISTERIA SANCTA», oficiar o para entrar en el coro, en el capítulo y en el refectorio ${ }^{45}$.

Su comportamiento debe adecuarse a los elementos sagrados y a los «ORDINES ECCLESIAE» para no arriesgarse a ser desautorizados como «REFUGANOS», es decir, como nicolaístas y simoníacos ${ }^{46}$, que son las connotaciones añadidas al significado de esta palabra visigótica que designa a los eclesiásticos errantes $\mathrm{y}$, en general, a los traidores hasta mediados del s. $\mathrm{XI}^{47}$. Las penas infamantes y la expulsión temporal de la Iglesia se esgrimen para forzarles al arrepentimiento y a regresar al «REGULAREM ORDINEM $»^{48}$.

42 Se renueva el precepto de abstinencia sexual que condena la convivencia con «MUJERES EXTRAN̄AS», a no ser la madre, tía o hermana en caso necesario, y con el deber de ir cubiertas con vestido monocolor o hábito religioso (Ver decr. III, 15 de Coyanza, Ed, García Gallo p. 22; y el cap. III [2] de ambos Concilios Compostelanos, Ed. MARTínez DíeZ p. 129). Los monjes ordenados presbíteros en 1071 reconocen el compromiso de no tener «IN IPSAS ECCLESIAS MULIERES EXTRANEAS» (RUIZ ASENCIO, J.M. Col...León, IV, 1180, p. 423).

43 Arraiga su caracterización por la condición de inermes que afectará tanto a clérigos como a monjes. «CLAUSTRA FRATRUM...INNOCENTUM ET SANCTE CASTIMONIE DILIGENTIUM» (Año 1042, según copia del último tercio del s. XI, HeRRERO DE LA FUENTE, M. Colección Diplomática..., II, cit, 473, pp. 134-135). Alfonso VI justifica en el año 1087 su conmiseración por los clérigos de Astorga con el argumento de «ESSE INERMES ASQUE DOMOS ET HABITATORES» (MUÑo7. Y Romero,T. Colección de Fueros Municipales y Cartas Pueblas, Madrid, 1972, pp. 321323).

44 Sobre el vestido talar, ver el decr. III, 12 de Coyanza, Ed. García GaLlo, p. 21, y los cap. I, 3 de ambos Concilios Compostelanos Ed. MARTÍNEZ DíEZ, p. 127. Sobre la tonsura y la barba rasurada, ver decr. III, 14 de Coyanza (Ibidem p. 21); y cap. II [4] del Concilio Compostelano de 1056 (Ibidem, p. 128).

45 Cap. VI [3] del Concilio Compostelano de 1063, Ed. MartíneZ DíeZ, p. 131.

46. «IPSI REFUGANTES QUI ORDINES ECCLESIAE DIMISERUNT ET UXORIBUS SE SOCIAVERUNT. DIMITTANT EAS ET IN CONFESSIONEM INTRENT) (Concilio Compostelano de 1056, cap. II [3] , Ed. MARTÍNEZ DÍEZ p. 129; y cap. III [1] del Concilio Compostelano de 1063, Ibidem, p. 128). Ver también cap. III [1] del Concilio de 1056, VI [1] del Concilio de 1063, Ibidem, pp. 128 y 130-131.

47 Los esfuerzos restauradores de Alfonso $\mathrm{V}$ para que respeten su adscripción al episcopado no impiden las fugas de monjes. En 1052 un presbítero custodiaba a los «MONAGOS ET REFUGANOS DE MANIBUS DE DOMNO ET PONTIFICE...» (Doc. cit. en la nota 18).

48 Quienes abandonando los «ORDINES ECCLESLAE» conviven con mujeres deben dejarlas y arrepentirse (cap. III [3] del Concilio Compostelano de 1056, Ed. MARTínEZ DiEZ p. 129). Todo clérigo que no custodie su «ORDINEM» queda separado de la Iglesia hasta que la penitencia lo restablezca en su seno (C. IV del Concilio Compostelano de 1063, Ed. MARTíNEZ DfEZ p. 129). El FUERO JUZGO seffala las penas para los que habiendo recibido «EL HABITO DE LA ORDEN» lo abandonen. $Y$ si de nuevo son «TORNADOS A LA ORDEN..., SEAN DIFAMADOS Y HAGAN MAS FUERTE PENITENCIA EN LOS MONASTERIOS» (Libro III, Tit. V, Ley III, Ed. R.A.E.).

$I^{\text {er }}$ Congreso de Historia de la Iglesia Hispania Sacra 51 (1999) 
Quienes abandonan su «ORDINATIONEM» después de haberla recibido en un monasterio son equiparados a «PERROS QUE VUELVEN AL VÓMITO» ${ }^{49}$, según la conocida perífrasis que denuncia reiteradamente en siglos posteriores las disfunciones del orden eclesiástico. Se instiga a que los monjes abandonen sus riquezas y practiquen sin hipocresía la pobreza y la caridad ${ }^{50}$, como forma de adecuación a las representaciones sociales que los agrupan con pobres y peregrinos ${ }^{51}$. La nueva sensibilidad cluniacense y la participación de clérigos «orientales» en la restauración de Iglesias castellanas ${ }^{52}$ propician la diferenciación entre monjes y clérigos. Sin embargo, y así lo indicamos en el primer epígrafe, perdura hasta el s. XII la permeabilidad entre ambas categorías funcionales. En la segunda mitad del $\mathrm{s}$. XI arraiga por influjo cluniacense la consideración de que el «ORDINEM MONASTICUM» es el mas perfecto de los órdenes eclesiásticos, debido a su aceptación de la «VIDA REGULAR» y su persistencia en la $«$ DISCIPLINA $»^{53}$.

La Iglesia episcopal y diocesana se restaura a partir de Coyanza mediante el impulso de la vida en común de clérigos y obispos en las sedes, y con la adscripción al episcopado de muchas Iglesias del sistema familiar o propio. Dicha vida en común se entiende inicialmente como uniformidad horaria para los actos litúrgicos, los rezos... y el uso colectivo del refectorio y del dormitorio ${ }^{54}$. Se determína el número, grado, conocimientos o edad que convienen a los distintos oficios, así como la calidad de las vestiduras y de los objetos litúrgi-

49 Concilio Compostelano de 1056, C. IV [2] , Ed, MARTtNeZ DízZ p. 130.

50 No pueden poseer nada propio, a no ser con licencia de su obispo o de su abad (decr. II [2] de la redacción conimbricense de Coyanza; Ed. GARcĺa GALLO, p. 18). En el abandono de bienes y en la práctica de la caridad profundizan ambos Concilios Compostelanos, en sus cap. IV, 1 y V, 1 respectivamente (Ed. MARTÍNEZ DÍEZ p. 130). «SANCTE CASTIMONIE DILIGENTIUM...ABEANT INTER SE IPSOS PACEM ET DILECTIONEM SEU ET CARITATEM NON FICTAM» (cit. en la n. 43).

5I Dichas representaciones abundan desde principios del segundo tercio del s. XI sin obviar en este período, a los «SACERDOTES» (Año 1055, RUlz ASENCiO, J.M. Col...León, IV, 1099, pp. 300-302).

52 La sede de Palencia por los obispos Poncio y Miro (ver la descripción retrospectiva de un doc. del afio 1059, ABAJo MARTín, cit., 9, 23-28). Y la Iglesia de Santa María del Puerto (Santoña) por el presbítero Paterno (Año 1047, SERRANO Y SANZ, M. «Cartulario de Santa María del Puerto». B.R.A.H., LXXIII-LXXX, 1918-1922, doc. $n^{\circ}$ VIII, pp. 429-431).

53 Resulta muy expresivo el documento apócrifo del año 1033 sobre la introducción de dicha observancia religiosa en el monasterio de Oña, que fué interpolado con posterioridad a 1073. ALAMO, J. del Colección diplomática de San Salvador de Oña, I, Madrid, 1950, 26, pp. 46-52.

54 El decr. I de Coyanza la califica de «VDA CANONICA» o de simple deber episcopal de guardar el ministerio eclesiástico en las sedes; según se lea la redacción conimbricense u ovetense (Ed. García Gallo p. 18). Ver Cap. I1 y I2 del Concilio Compostelano de 1056 Ed. MarTíneZ DIEZ, p. 127. 
$\cos ^{55}$. Desde entonces, y con lentitud, se abre paso la observancia de una «REGULA CANONICA» ${ }^{56}$.

Los presbíteros y diáconos están obligados a cumplir los decretos de Coyanza so pena de privación del «GRADUS ECCLESIASTICO» ${ }^{57}$. Si abandonan la «CAUSA ECCLESIASTICA», no respetan su «ORDINEM» o viven ilícitamente con mujeres, son expulsados «CUM LAICIS PAREM ORDINEM» hasta su arrepentimiento ${ }^{58}$. Los clérigos quedan liberados de la obligación de prestar servicio a los laicos y de aceptar su potestad a no ser por consentimiento propio o mandato episcopal ${ }^{59}$. Los órdenes eclesiásticos se cierran a mediados del s. XI frente al secular, que se instaura para definir su estilo de vida y ámbitos de competencia. No existe, de momento, una dualidad radical sustentada en dos funciones, sino tan solo una distinción y separación de competencias y de conductas.

Los laicos carecen de ordenación hasta el sínodo-imperial de Coyanza, que les confiere cohesión como «SECULARIS ORDO» ${ }^{60}$. Fueron investidos entonces de responsabilidades negativas y positivas hacia los elementos y espacios sagrados, y hacia las personas y bienes eclesiásticos. Se les prohíbe convivir con sus mujeres en los «DEXTROS $»^{61}$, y se les apremia a renunciar a la «POTESTATEM» sobre clérigos e Iglesias, o a la «DELEGATIONEM» sobre las cosas o causas de estas ${ }^{62}$. Obstaculizan, de esta manera, su permanencia o caída «IN LAICAVILIS» o en el «SECULARE DOMINIUM»63.

55 Cap. I [1] de ambos Concilios compostelanos; I [3] , I [4] y I [5] del Concilio Compostelano de 1056 (Ed. MARTfNeZ DíeZ, p. 127). Decr. III [5] , [6] y [7] de Coyanza (Ed. GARCía GALLo, p. 20).

s6 Cap. I [1] del Concilio Compostelano de 1063 (Ed. MARTínEZ DfEZ, p. 127). En 1082 existe en la sede leonesa un «PRAEPOSITUS CANONICORUM» (RUIZ ASENCIO, J.M. Col...León, IV, 1126, pp. 502-504),

57 Decr. III [18] de ambas redacciones Ed. GaRCfa Gallo p. 23.

58 Cap. IV y VI 1 del Concilio Compostelano de 1063 (Ed. MARTínez DífZ, pp. 129-131). Indica que los canónigos con mujer legítima deben abandonar la causa eclesística y «CUM LAICIS PAREM ORDINEM TENEANT'.

59 Decr. Ш [2] de ambas redac. de Coyanza (GARCía GaLLo, cit. p. 19).

60 [SANCTIO] de la versión ovetense (Ed. GARCía GALLO p. 30). El «LAICUS» se contrapone a los consagrados a Dios, generalmente en las copias escritas a partir de mediados del s. XI (Año 1042, HERRERO DE LA FUENTE, cit., 473, pp. 134-138). El Fuero Juzgo refleja dicha oposición entre los «LEGOS» y los «ORDENADOS» por excelencia (Libro II, Tit. I, Ley VII. Ed. RAE).

61 Decr. [16] de ambas redac. de Coyanza (Ed. GARCf́A GALLO, p. 22) y cap. LII, [1] del Concilio Compostelano de 1056 (Ed. MARTÍNEZ DíEZ, p. 128).

62 Decr. III, 2 de ambas redacciones de Coyanza (Ed. GarCf́a GaLlo, p. 19). Concilio Compostelano de 1056, cap. I [5] (Ed. MARTf́NEZ DíEZ, p. 127).

63 Año 1057. S. García LaRragueta, Colección de documentos de la Catedral de Oviedo, Oviedo, 1962, 59, pp. 186-187.

$f^{\text {It }}$ Congreso de Historia de la Iglesia Hispania Sacra 51 (1999) 
Es propio de los laicos portar armas «SAECULARIA» o «BELLICIS», ejercer la «SAECULARIA POTESTATE», ocuparse «IN NEGOTIIS SAECULARIBUS» y tener mujeres legítimamente ${ }^{64}$. De forma tácita, se circunscribe el ámbito de su actuación a la guerra, al ejercicio del poder, a la acumulación de riqueza y a la procreación. Naturalmente todo ello está regulado por los valores y la moral cristiana que modela las relaciones matrimoniales, la sexualidad...65; y reprende la violencia y la rapacidad de los poderosos, o el enriquecimiento sin distribución de bienes hacia los pobres y hacia las instituciones eclesiásticas. La taxonomía social de la segunda mitad de la centuria contrapone frecuentemente los poderosos a los débiles, los gobernantes a los gobernados, y los ricos a los pobres.

Nuevos criterios de clasificación social individualizan a los integrantes de cada orden a partir de mediados del s. XI. Entre los eclesiásticos adquiere una relevancia especial el grado de cumplimiento de sus propias normas y leyes ${ }^{66}$, además de la coherencia con su calidad de castos, inermes y pobres. Los obispos y abades acusados por simonía ${ }^{67}$ son coetáneos o muy próximos a quienes alcanzaron la santidad: S. Albito de León, S. Ordoño de Astorga, S. Fagildo del monasterio compostelano de Antealtares, S. Iñigo de Oña o Santo Domingo de Silos. Sobre esta situación incide el rito y costumbres romanas que se introducen en Castilla y León a partir de 1075. Los clérigos de algunas sedes episcopales fueron especialmente reacios a abandonar el «MORE LAICORUM» y a cumplir con la «DISCIPLINA ECLESIASTICA» ${ }^{68}$.

64 Reciben estas denominaciones cuando son vedadas a los eclesiásticos. Ver el decr. III [13] de Coyanza (Ed. Garcla Gallo p. 21) y los cap. II [4] y II [3] de ambos Concilios Compostelanos (Ed. MARTINEZ DfEZ p. 128); y el cap. IV [1] del Concilio Compostelano de 1056 (Ibidem, p. 130).

65 Los cristianos no pueden tener dos mujeres, ni contraer matrimonio entre consanguíneos hasta el séptimo grado. Se reprende a los adúlteros, incestuosos y a quienes no legitiman su retación con una mujer o la abandonan.

66 Sean éstas romanas o no. El obispo y los canónigos de Palencia «CUIUS CUMQUE CONDITIONES AU'T OFFICII VEL LEGIS MORATOR FUERIT» son considerados «DOMINI" de los babitantes de la ciudad y de sus alrededores, a tenor de un documento interpolado del año 1059 (ABAJO MARTIN, cit, 9, pp. 23-28).

67 El obispo Nuño de Oca y el abad Roberto de Sahagún fueron considerados simoníacos en Roma (D. MANSILLA, La documentación pontificia hasta Inocencio III (965-1216), Roma, 1955, doc. 8 y 19 pp. 15-16 y 32-33).

68 En la sede de Astorga «MAXIMA PARS CLERICORUM CONVERSABANTUR FORO MORE LAICORUM» (Año 1087, MUÑoz Y ROMERO, cit. pp. 321-323). Cuando Diego Gelmírez ocupó la sede compostelana en los umbrales del s. XII, los clérigos existentes eran «UNOS PATANES QUE PRACTICAMENTE NO ESTABAN SOMETIDOS A DISCIPLINA ECLESIASTICA ALGUNA» (Historia Compostelana, Libro Y, cap. XX Ed. E. FALQUE, cit. p. 110).

$\mathrm{I}^{\mathrm{er}}$ Congreso de Historia de la Iglesia Hispania Sacra 51 (1999) 